\title{
Multidrug resistant Enterobacteriaceae from blood stream infections at a tertiary care hospital of Eastern Nepal
}

\author{
Ajay Yadav ( $\nabla$ yajay427@gmail.com ) \\ National Medical College and Teaching Hospital, Nepal \\ Narayan Raj Bhattarai \\ BP Koirala Institute of Health Sciences \\ Basudha Khanal \\ BP Koirala Institute of Health Sciences
}

\author{
Research \\ Keywords: AmpC, Enterobacteriaceae, ESBL, metallo-beta-lactamase, multidrug resistant, ten disk method \\ Posted Date: July 28th, 2020 \\ DOI: https://doi.org/10.21203/rs.3.rs-44323/v1 \\ License: (c) (1) This work is licensed under a Creative Commons Attribution 4.0 International License. Read Full License
}

Version of Record: A version of this preprint was published at Journal of Bio Innovation on March 30th, 2021. See the published version at https://doi.org/10.46344/JBIN0.2021.v10i02.13. 


\section{Abstract \\ Background}

Blood stream infection (BSI) is one of the major causes of morbidity and mortality worldwide. It has poses significant challenge to the clinicians and clinical microbiologists alike. Therefore its accurate diagnosis, isolation and identification of causative agents with appropriate antibiotics is required. This study is aimed to find out resistance pattern, e.g. extended-spectrum-beta-lactamase (ESBL), AmpC, K1, carbapenemase and metallo- $\beta$-lactamase (MBL) among isolates obtained from BSI.

\section{Methods}

A cross-sectional study was conducted in the Department of Microbiology, BPKIHS from 1st September 2014 to 31 st August 2015. Isolates were screened for $\mathrm{ESBL}, \mathrm{AmpC}, \mathrm{K} 1$, and carbapenemase production by ten disk method. Confirmation for ESBL was done phenotypically by using combined disk method recommended by CLSI, AmpC sterile disk method for AmpC and $\mathrm{K} 1$ by combined disk method. Metallo-beta-lactamase (MBL) production was detected by imipenem-ethylene-diamine-tetra acetic acid double disk synergy test.

\section{Results}

A total of 11,264 blood samples were collected from the patients suspected of Blood Stream Infection. Of these isolates, 192 (1.70\%) were Enterobacteriaceae. Among them, 94 (49\%) were ESBL, 51 (26.5\%) were carbapenemase and 10 (5\%) were AmpC producers. Of 51 carbapenemase producers, 22 (11.5\%) were MBL producers. None of the isolates were found to produce K1 $\beta$-lactamase. A total of $64(33.4 \%)$ isolates were MDR.

\section{Conclusion}

MDR Enterobacteriaceae is found to be prevalent in our set up as important cause of BSI.

\section{Introduction}

Blood stream infection (BSI) is one of the major causes of morbidity and mortality worldwide. On one hand advancement in the patient care services such as widespread use of indwelling devices, complicated surgery and prolonged hospitalization have emerged as important risk factors for occurrence of BSI. On the other hand involvement of the resistant microorganisms as etiological agents of BSI has posed significant challenge to the clinicians and clinical microbiologists alike. Accurate diagnosis of BSI, timely isolation and identification of the causative agents and determination of their antimicrobial susceptibility are crucial, as effective management depends on the selection and timely administration of the most appropriate antimicrobial agent [1]

Over the recent years, the problem of multidrug resistant Enterobacteriaceae has accelerated dramatically and occupies the third most leading cause of BSI in several settings [2]. The escalating burden of multidrug resistance in Enterobacteriaceae is largely due to production of beta lactamase and subset of beta lactamases, which are enzymes that bind, deactivate the different types of beta lactam antibiotics and confer broad resistance to them [3].

Despite the attempts of antibacterial stewardship and rigorous endeavor against MDR bacteria, its incidence is not decreasing [4]. This is a worrying public health issue as the infections caused by such enzymes producing bacteria are associated with higher morbidity and mortality. It is a threat to developing countries like Nepal who has greater economic burden as these enzymes can be carried on bacterial chromosomes or may be plasmid mediated and has potential to move between bacterial populations. A comprehensive and cost effective approach is essential for the early identification of the resistant strains and rational institution of therapy.

This study was, therefore, undertaken with an aim to determine ESBL, AmpC, K1, carbapenemase and metallo- $\beta$-lactamase (MBL) producing Enterobacteriaceae isolated from blood stream infections (BSI) at BPKIHS with special reference to the detection of Multidrug resistant Enterobacteriaceae by ten disk approach.

\section{Methods}

Setting of this study was Department of Microbiology, BPKIHS. A total of 11,264 blood specimens were submitted to Department of Microbiology for culture. Identification and sensitivity of the isolates were analyzed as per standard microbiological procedures [5].

\subsection{Antimicrobial susceptibility testing}

Antimicrobial susceptibility test of all the isolates was performed on Mueller Hinton agar (MHA) (Hi Media, India) by the standard disk diffusion technique of Kirby-Bauer method and interpreted as per Clinical and Laboratory Standards Institute (CLSI) recommendations [6].

\subsection{Detection of ESBL}

\subsubsection{ESBL screening test}


ESBL screening was done as per CLSI recommendations. Isolate showing inhibition zone of $\leq 27$ for cefotaxime (CTX) (30 $\mu \mathrm{g})$ and $\leq 22 \mathrm{for}$ ceftazidime (CAZ) $(30 \mu \mathrm{g})$ was taken for ESBL confirmation [6].

\subsubsection{ESBL confirmatory test}

The antimicrobial disks (HI Media, Mumbai, India) used were CTX $(30 \mu \mathrm{g}), \mathrm{CAZ}(30 \mu \mathrm{g})$, cefotaxime-clavulanic acid (CEC) $(30 \mu \mathrm{g} / 10 \mu \mathrm{g})$ and ceftazidimeclavulanic acid (CAC) $(30 \mu \mathrm{g} / 10 \mu \mathrm{g})$. Each disk was kept at least $20 \mathrm{~mm}$ apart, center to center. Isolates resistant to CTX and CAZ but sensitive to CEC and CAC with enhanced $\mathrm{ZOI} \geq 5 \mathrm{~mm}$ was confirmed as ESBL producers [6].

\subsection{Detection of AmpC}

\subsubsection{Amp C screening test}

For this, antimicrobial disks used to screen AmpC $\beta$-lactamase were cefoxitin (CX) (30 $\mu \mathrm{g})$ and cefepime (CPM) (30 $\mu \mathrm{g})$. Isolates resistant to CX (ZOI $\leq 18 \mathrm{~mm})$ but sensitive to $\mathrm{CPM}(\mathrm{ZOI} \geq 18 \mathrm{~mm})$ indicates $\mathrm{AmpC}$ producers [7].

\subsubsection{Amp C confirmatory tests}

\subsubsection{Amp C sterile disk test}

Confirmation was done by performing AmpC sterile disk test. MHA plate was inoculated with Escherichia coli ATCC 25922. CX disk was placed on MHA plate. Adjacent to it, a sterile disk was placed. On the sterile disk, isolated strain was inoculated several times. After incubation, flattened or intended zone produced around the $\mathrm{CX}$ disk was confirmed as AmpC $\beta$-lactamase production [8].

\subsubsection{Modified Hodge Test}

Escherichia coli ATCC 25922 was inoculated on MHA plate. CX disk was placed at the center of plate with the help of sterile forcep. Isolated strain was inoculated perpendicular to the $\mathrm{CX}$ disk and was incubated overnight aerobically at $35^{\circ} \mathrm{C}$. Presence of clover leaf appearance at the streaking line of the isolated strain was interpreted as AmpC producers [9].

\subsection{Detection of K1}

For the detection of $\mathrm{K} 1 \beta$-lactamase, the antimicrobials used were aztreonam (AT) $(30 \mu \mathrm{g})$, ceftriaxone (CTR) $(30 \mu \mathrm{g}), \mathrm{CTX}(30 \mu \mathrm{g})$ and CAZ (30 $\mu \mathrm{g})$. A strain was considered $\mathrm{K} 1 \beta$-lactamase producer if it was resistant to AT (ZOI $\leq 27 \mathrm{~mm})$ and CTR (ZOI $\leq 25 \mathrm{~mm})$ and sensitive to CTX (ZOI $\geq 26 \mathrm{~mm})$ and CAZ (ZOI $\geq 21 \mathrm{~mm})[10,11]$.

\subsection{Detection of Carbapenemase}

For the screening of carbapenemase, isolates should be resistant to ertapenem (ETP) $(10 \mu \mathrm{g})(\mathrm{ZOI} \leq 22 \mathrm{~mm})$ and sensitive to imipenem (IPM) (10 $\mu \mathrm{g})(\mathrm{ZOI} \geq$ $23 \mathrm{~mm}$ ). Besides this, rest of the antimicrobials should be resistant for the isolates to be carbapenemase producers [12].

2.6 Metallo $\beta$-lactamase (MBL)

\subsubsection{MBL screening test}

The isolates were interpreted as MBL producers when ZOI for CAZ $(30 \mu \mathrm{g})$ was $\leq 18 \mathrm{~mm}$ [13].

\subsubsection{MBL confirmation tests}

\subsubsection{Double disk synergy test}

For double disk synergy test, anhydrous ethylene diamine tetra acetic acid (EDTA) disk of concentration $1.5 \mathrm{mg} / \mathrm{disk}(0.5 \mathrm{~mol}$.) and imipenem (IPM) (10 $\mu \mathrm{g})$ were used. EDTA-IPM disks were placed at a distance of $10 \mathrm{~mm}$ apart. Enhanced ZOI showing synergistic effect between the two disks was considered as indication of MBL productions [14]

\subsubsection{Modified Hodge Test (MHT)}

For this, ATCC Escherichia coli was inoculated on MHA plate. IPM disk was placed at the center of MHA plate with the help of sterile forcep. Isolated strain was inoculated perpendicular to the IPM disk. Following incubation, appearance of a clover leaf at the streaking line of the isolated strain was confirmed as MBL producers [15].

\subsection{Definition of MDR}

Multidrug resistance (MDR) is the resistance offered by bacteria to three or more different antibiotic classes [16].

\section{Results}

During the study period, a total of 11,264 blood samples were collected from the patients subjected to Blood Stream Infections. Of these isolates, 192 (1.70\%) were Enterobacteriaceae comprising of Escherichia coli 95 (49.48\%), Klebsiella pneumoniae 45 (23.44\%), Enterobacter aerogenes 27 (14.06\%), Citrobacter freundii 6 (3.13\%), Citrobacter koseri 6 (3.13\%), Proteus vulgaris 3 (1.56\%), Salmonella Typhi 3 (1.56\%), Enterobacter cloacae 2 (1.04\%), Proteus mirabilis 2 (1.04\%), Klebsiella oxytoca 1 (0.52\%), Morganella morganii 1 (0.52\%) and Salmonella Paratyphi A 1 (0.52\%). (Fig. 1) 
Figure 1: Frequency of Enterobacteriaceae isolates $(n=192)$

$113(59 \%)$ isolates were obtained from male patients and 79 (41\%) from female patients. Majority of the isolates were from age group < 10 years $(71 ; 37 \%)$, followed by group $50-89$ years $(24 \%)$ and $20-29$ years $(13 \%)$.

\subsection{Frequency of $\beta$-lactamases producers}

Results of different types of $\beta$-lactamases produced by Enterobacteriaceae are given in Fig. 2. ESBL was the most common $\beta$-lactamase producers 94 (49\%), followed by carbapenemase producers 51 (26.5\%), MBL producers $22(11.5 \%)$ and AmpC producers $10(5 \%)$. In none of

the isolates $\mathrm{K} 1 \beta$-lactamase has been detected.

Among IPD Enterobacteriaceae isolates, ESBL producers were 33 (17\%), AmpC 4 (2\%), carbapenemase 24 (12.5\%) and MBL 14 (7\%). Similarly from OPD, 61 (32\%) were ESBL producers, 6 (3\%) AmpC producers, 27 (14\%) carbapenemase producers and 8 (41\%) MBL producers. Comparing $\beta$-lactamases producers among both IPD and OPD, ESBL, AmpC and carbapenemase producers were more in OPD but MBL producers were more in IPD.

\section{2 $\beta$-lactamase producers among Enterobacteriaceae}

Among 192 Enterobacteriaceae isolates, the number of ESBL producers were 94 (49\%), AmpC 10 (5\%), carbapenemase 51 (26.5\%) and MBL 22 (11.5\%). Among 95 Escherichia coli isolates, 60 (92\%) were ESBL producers, 2 were AmpC producers, 23 were carbapenemase producers and 3 were MBL producers. Among isolates, only one Klebsiella oxytoca was isolated which was ESBL producers. The isolates that were non $\beta$-lactamase producers were Morganella morganii (1) and P. mirabilis (2). After ESBL, carbapenemase (51) was the second most $\beta$-lactamase producers and here also Escherichia coli was (23) predominant in number followed by K. pneumoniae (18). Among MBL producers, K. pneumoniae (13) were the most in numbers followed by E. coli (3), E. aerogenes (3), C. koseri (2) and C. freundii (1). Among

Table 1

$\beta$-lactamases producers among Enterobacteriaceae

\begin{tabular}{|lllll|}
\hline Enterobacteriaceae $(\mathbf{n = 1 9 2 )}$ & $\operatorname{ESBL}(\mathbf{n = 9 4 )}$ & $\operatorname{AmpC}(\mathbf{n = 1 0})$ & Carbapenemase $(\mathbf{n = 5 1})$ & MBL $(\mathbf{n}=\mathbf{2 2})$ \\
\hline E. coli & 60 & 2 & 23 & 3 \\
\hline K. pneumoniae & 19 & 2 & 18 & 13 \\
\hline E. aerogenes & 8 & 2 & 5 & 3 \\
\hline C. freundii & 2 & 2 & 1 & 1 \\
\hline P. vulgaris & 2 & 0 & 1 & 0 \\
\hline K. oxytoca & 1 & 0 & 0 & 2 \\
\hline C. koseri & 1 & 1 & 3 & 0 \\
\hline E. cloacae & 1 & 0 & 0 & 0 \\
\hline S. Typhi & 0 & 1 & 0 & 0 \\
\hline S. Paratyphi A & 0 & 0 & 0 & 0 \\
\hline M. morganii & 0 & 0 & 0 & 0 \\
\hline
\end{tabular}

AmpC producers, two from each E.coli, $K$ pneumoniae, E. aerogenes and $C$. freundii were isolated. Similarly, different types of $\beta$-lactamases producers were elucidated in Table 1.

\subsection{Frequency of AmpC producers}

Equal number of K. pneumoniae, E. aerogenes, C. freundii and E. coli were found to be AmpC producers, and one each of $S$. Typhi and $C$. koseri. Of the total AmpC producers (10), five were positive for AmpC confirmatory test. (Table 2) 
AmpC producers $(n=10)$

\subsection{Frequency of MBL producers}

\begin{tabular}{|llll|}
\hline Isolates $(\mathrm{n}=10)$ & AmpC screening test $(\mathrm{n}=10)$ & AmpC confirmatory test $(\mathrm{n}=\mathbf{5})$ & Department \\
\hline K. pneumoniae $(\mathrm{n}=1)$ & Positive & Positive & IPD \\
\hline K. pneumoniae $(\mathrm{n}=1)$ & Positive & Negative & OPD \\
\hline E. aerogenes $(\mathrm{n}=2)$ & Positive & Positive & OPD \\
C. freundii $(\mathrm{n}=1)$ & Positive & Positive & IPD \\
\hline C. freundii $(\mathrm{n}=1)$ & Positive & Negative & OPD \\
\hline E. coli $(\mathrm{n}=1)$ & Positive & Positive & OPD \\
\hline E. coli $(\mathrm{n}=1)$ & Positive & Negative & OPD \\
\hline S. Typhi $(\mathrm{n}=1)$ & Positive & Negative & IPD \\
\hline C. koseri $(\mathrm{n}=1)$ & Positive & Positive & IPD \\
\hline
\end{tabular}

Among the isolates, 22 (11.5\%) were found to be MBL producers, which was elucidated in Fig. 4. Among these, K. pneumoniae (13, 59.1\%) was found to be most MBL producers followed by $E$. coli $(3,13.6 \%)$, E. aerogenes $(3,13.6 \%)$, and $C$. koseri $(2,9.1 \%)$. Among the isolates, only one $C$. freundii was isolated which was MBL producers. (Fig. 3)

\subsection{Antibiotic sensitivity pattern of Enterobacteriaceae}

Among 192 isolates, most were resistant to third generation cephalosporins, cefotaxime $(141 ; 73.40 \%)$, ceftazidime (139; 72.40\%) and ceftriaxone (105; $54.70 \%$ ). Talking about sensitivity pattern with cefepime (fourth generation cephalosporin), similar pattern, i.e. (113; $58.80 \%)$ was observed as those of sensitivity pattern with third generation cephalosporins. Most of the isolates of $E$. aerogenes $(17 ; 63 \%)$ and $C$. freundii $(4 ; 67 \%)$ were sensitive to cefepime. All three S. Typhi were sensitive to cefepime. Regarding monobactam, $77(40 \%)$ isolates were found sensitive and the number of resistant isolates were equal to the sensitive isolates. Among carbapenems, 22 (11.5\%) isolates were resistant to imipenem and 51 (26.5\%) isolates were found resistant to ertapenem. Most of the isolates $(164 ; 85.4 \%)$ were sensitive to imipenem. Almost $99 \%$ of the isolates were found sensitive to tigecycline. (Table 3 )

Table 3

Antibiotic sensitivity pattern of the Enterobacteriaceae

\begin{tabular}{|llll|}
\hline Antibiotic used & Sensitive (\%) & Resistance (\%) & Intermediate (\%) \\
\hline Cefotaxime & 25.50 & 73.40 & 1.10 \\
\hline Ceftazidime & 23.96 & 72.40 & 3.64 \\
\hline Ceftriaxone & 42.70 & 54.70 & 2.60 \\
Cefotaxime-clavulanic acid & 71.90 & 28.10 & 0.00 \\
\hline Ceftazidime-clavulanic acid & 68.30 & 31.70 & 0.00 \\
\hline Cefoxitin & 41.70 & 41.70 & 16.6 \\
\hline Cefepime & 39.60 & 58.9 & 1.50 \\
\hline Aztreonam & 40.10 & 56.25 & 3.65 \\
\hline Ertapenem & 66.63 & 26.56 & 7.81 \\
\hline Imipenem & 85.40 & 11.50 & 3.10 \\
\hline Colistin & 96.40 & 3.10 & 0.50 \\
\hline Tigecycline & 98.96 & 0.00 & 1.04 \\
\hline
\end{tabular}

\subsection{MDR Enterobacteriaceae}

Among 192 isolates of Enterobacteriaceae, 64 (33.3\%) isolates were found to be multidrug resistant. Escherichia coli (26) was found to be the leading one among the MDR isolates, followed by Klebsiella pneumoniae (21), Enterobacter aerogenes (8), Citrobacter koseri (3), Citrobacter cloacae (2) and one each of Citrobacter freundii, Klebsiella oxytoca, Proteus vulgaris, and Salmonella Paratythi A. (Fig. 4)

\subsection{Enterobacteriaceae resistance to all beta-lactam drugs}

Among 192 Enterobacteriaceae, 22 isolates were resistant to all $\beta$-lactam drugs - extended spectrum cephalosporins, cephamycins, monobactum and carbapenems. (Fig. 5)

A total of 22 isolates were found to be resistant to all $\beta$-lactam drugs. Almost all were isolated from inpatients $(21 ; 95.5 \%)$ and only one from outpatient. All these were metallo-B-lactamase producers $(\mathrm{n}=22)$, among which $13(59 \%)$ were K. pneumoniae, $3(13.6 \%)$ each were $E$. coli and $E$. aerogenes, $2(9 \%)$ were $C$. 
koseri and 1 (4.5\%) was C. freundii. Distribution among different departments of origins were as follows; 8 (36\%) from ER, 4 (18\%) from ICU, 3 (13.6\%) from DICU, 2 (9\%) each from nursery and NICU and 1 (4.5\%) from medicine outpatient department.

\subsection{Distribution of Enterobacteriaceae among different wards}

Table 4 shows distribution of Enterobacteriaceae among different wards. Escherichia coli (30.2\%) was found to be more common among the isolates in ER, followed by Klebsiella pneumoniae (8.3\%) and E. aerogenes (7.3\%). In ICU, K. pneumoniae was the leading one, followed by E. coli and E. aerogenes. In PICU and DICU, only one isolate, $K$ pneumoniae, in

each ward was isolated. Two isolates were from NICU, K. pneumoniae and C. koseri.

Table 4

Distribution of Enterobacteriaceae among different wards $(\mathrm{n}=192)$

\begin{tabular}{|c|c|c|c|c|c|c|c|c|c|c|c|c|c|}
\hline Wards & $\begin{array}{l}E . \\
\text { coli }\end{array}$ & $\begin{array}{l}\text { K. } \\
\text { pneumoniae }\end{array}$ & $\begin{array}{l}\text { K. } \\
\text { oxytoca }\end{array}$ & $\begin{array}{l}\text { C. } \\
\text { freundii }\end{array}$ & $\begin{array}{l}\text { C. } \\
\text { koseri }\end{array}$ & $\begin{array}{l}\text { M. } \\
\text { morganii }\end{array}$ & $\begin{array}{l}\text { E. } \\
\text { aerogenes }\end{array}$ & $\begin{array}{l}\text { E. } \\
\text { cloacae }\end{array}$ & $\begin{array}{l}P . \\
\text { vulgaris }\end{array}$ & $\begin{array}{l}P \\
\text { mirabilis }\end{array}$ & $\begin{array}{l}S . \\
\text { Typhi }\end{array}$ & $\begin{array}{l}\text { S. } \\
\text { Paratyphi } \\
\text { A }\end{array}$ & Tot \\
\hline DICU & 0 & 0 & 0 & 0 & 1 & 0 & 0 & 0 & 0 & 0 & 0 & 0 & 1 \\
\hline ER & 58 & 16 & 1 & 3 & 2 & 1 & 14 & 2 & 2 & 1 & 1 & 1 & 102 \\
\hline GOPD & 8 & 4 & 0 & 0 & 0 & 0 & 0 & 0 & 0 & 0 & 1 & 0 & 13 \\
\hline $\begin{array}{l}\text { Obs \& } \\
\text { Gynae }\end{array}$ & 5 & 0 & 0 & 0 & 0 & 0 & 1 & 0 & 0 & 0 & 0 & 0 & 6 \\
\hline ICU & 4 & 6 & 0 & 0 & 2 & 0 & 0 & 0 & 0 & 0 & 0 & 0 & 12 \\
\hline Medicine & 4 & 3 & 0 & 2 & 1 & 0 & 3 & 0 & 1 & 1 & 1 & 0 & 16 \\
\hline NICU & 0 & 1 & 0 & 0 & 0 & 0 & 1 & 0 & 0 & 0 & 0 & 0 & 2 \\
\hline Nursery & 4 & 8 & 0 & 0 & 0 & 0 & 1 & 0 & 0 & 0 & 0 & 0 & 13 \\
\hline Orthopedics & 0 & 0 & 0 & 0 & 0 & 0 & 1 & 0 & 0 & 0 & 0 & 0 & 1 \\
\hline Paeditrics & 8 & 5 & 0 & 1 & 0 & 0 & 3 & 0 & 0 & 0 & 0 & 0 & 17 \\
\hline Surgery & 4 & 1 & 0 & 0 & 0 & 0 & 3 & 0 & 0 & 0 & 0 & 0 & 8 \\
\hline PICU & 0 & 1 & 0 & 0 & 0 & 0 & 0 & 0 & 0 & 0 & 0 & 0 & 1 \\
\hline Total & 95 & 45 & 1 & 6 & 6 & 1 & 27 & 2 & 3 & 2 & 3 & 1 & 192 \\
\hline
\end{tabular}

\section{Discussion}

To our knowledge, this type study is conducted first time in Nepal with an aim to evaluate multidrug resistance pattern in Enterobacteriaceae isolated from BSIs with special reference to ten disk method, and to guide the clinicians with the most appropriate antibiotics against those pathogens. MDR pattern is most commonly seen in Gram-negative bacteria compared to Gram-positive bacteria (GPB). Particularly resistance in GNB is of great importance as there is a dearth of novel antibiotics directed against these organisms [16]. Among the antimicrobials used, $\beta$-lactams are the most commonly used therapeutic class of antimicrobials for treatment of bacterial infections because of their broad antibacterial spectrum and excellent safety profile [17].

In our study, the frequency of BSIs by Enterobacteriaceae was found to be 1.7\% (192/11,264) out of total blood culture positive 17.3\% (1948/11,264). A similar study conducted by Abdallah et al in 2015 in Egyptian patients and in Nepal by Joshy M Easow et al in 2010 where the number of Enterobacteriaceae isolated found to be 94 and 96 respectively, which was less as compared to our study [17, 18].

All together 192 Enterobacteriaceae isolates were included in this study. Among them, most of the isolates (115; $60 \%)$ were obtained from outpatient departments, while 77 (40\%) were obtained from inpatient departments. The number of isolates were more from outpatient departments compared to inpatient, male to female ratio was found to be 1.4:1 which was found similar to the finding of study conducted in the western region of Nepal by Joshy $\mathrm{M}$ Easow et al in 2010.[18] The etiological agents of BSIs caused by Enterobacteriaceae are listed in Table 1. Majority of the isolates were Escherichia coli 49.5\% as compared to the other isolates. Similar results have been documented by Abdallah et al [17]. In a study conducted by Joshy M Easow et al 2010, Klebsiella pneumoniae 13.5\% was found to be the predominant isolates causing BSIs, a finding different as compared to our study [18]. Isolation of Escherichia coli (66), Enterobacter aerogenes (14), Enterobacter cloacae (2), Salmonella Typhi (2) and Proteus vulgaris (2) were more from OPD as compared to inpatient departments.

In Enterobacteriaceae, $\beta$-lactamase production remains the most important mediator of $\beta$-lactam resistance [17]. The rates of antimicrobial drug resistance and particularly of multiple drug resistance are increasing among Enterobacteriaceae, thus limiting the armamentarium of potentially active antimicrobial agents [19].

In the present study, $49 \%$ ESBL were isolated. Among them, $64 \%$ were Escherichia coli followed by K. pneumoniae $20 \%$. In a study conducted by Shrestha et al at BPKIHS in the year 2007, prevalence of ESBL among the clinical isolates of pyogenic infections were reported as isolates are- $E$. coli, K. pneumoniae, $P$. mirabilis, Enterobacter species and Citrobacter species 53\%,14.8\%,12.9\%,5.5\% and 5.5\% respectively [20]. Another study conducted at BPKIHS by Abhilasha 
Sharma in the year 2012, found the prevalence of ESBL as E. coli $73 \%$, K. pneumoniae $60.5 \%$, Enterobacter species $46 \%$ and Citrobacterspecies $25 \%$. These data suggest that at our institution E. coli remains as the most frequent ESBL producers followed by K. pneumoniae, Enterobacter species and Citrobacter species. Many studies have been conducted in Nepal regarding prevalence of ESBLs. In a study conducted by Raut et al. 2015 at Manipal Teaching Hospital, Pokhara, the prevalence of ESBL in E. coli was found to be $81.6 \%$ and in K. pneumoniae (4.1\%) which is similar to our study [21]. A comparable results were reported by Poudyal et al. 2011, i.e. the predominance of ESBL E. coli $80 \%$ as compared to K. pneumoniae 5.8\% [22]. Emergence of ESBL may be due to widespread use of third-generation cephalosporins and aztreonam which is believed to be the major cause of mutations in TEM and SHV enzymes [23].

There has been a paucity of data about the prevalence of AmpC producing strains in Nepal, and very little information is available about its distribution in different age groups. In the present study, 10(5\%) isolates were AmpC positive. Out of these, 5 isolates were positive by confirmatory test (Table 2 ). The rate of AmpC production was less compared to other study done in Nepal suggesting lesser spectrum of resistance among our isolates [24].

In the present study, K1 $\beta$-lactamase was not found in any of the isolates. This enzyme was first detected in Klebsiella pneumoniae. It is also detected in Klebsiella oxytoca $[25,26]$. In the present study, 46 (24\%) Klebsiella species were isolated. Among these, 40 were positive for $\beta$-lactamases- ESBL 20 , AmpC 2 , carbapenemase 18. Among 18 carbapenemase producers 13 were MBL producers, but all were negative for K $1 \beta$-lactamase. Carbapenemase producers in this study was found to be 51 (26.5\%). It was further tested by performing sensitivity test with tigecycline and colistin. Among the isolates, 190 ( $99 \%)$ were sensitive to tigecycline and 185 (96\%) were found sensitive to colistin. Two E. coli were found to have intermediate susceptibility to tigecycline. Talking about activity of colistin, six isolates were resistant to colistin, one had intermediate susceptibility. In this study, Tigecycline was very active and appears to be an excellent option compared to colistin for treatment of infections caused by these multidrug-resistant Enterobacteriaceae [27, 28].

The production of MBLs in strains largely limits therapeutic options. In screening of MBL, $22(11.5 \%)$ isolates were found to be imipenem resistant, whereas $51(26.5 \%)$ were resistant to ertapenem. The MBL producing Klebsiella species in the present study was found to be higher in number than that shown by Shrestha et al. [29]. In a study carried out by Vinod Kumar et al., $20 \%$ resistance to imipenem and $17 \%$ rate of MBL production was reported. Similarly, Kamble et al., reported $20 \% \mathrm{MBL}$ production [30].

In most centers $\beta$-lactamase production is not routinely tested which ultimately results in the dissemination of $\beta$-lactamase producing strains in hospitals, and it remains undetected for longer periods. Irrational use of antimicrobials leads to escalate increased percentage of $\beta$-lactamase production. Therefore, irrational use of antibiotics should be done as little as possible and specific therapeutic antibiotics should be used for short period as suggested by Singh et al. [30].

\section{Antimicrobial susceptibility pattem}

Antimicrobial susceptibility profile of total 192 isolates and selectively of the isolates producing $\beta$-lactamases (ESBL, AmpC, carbapenemase and metallobeta-lactamase) showed a high degree of resistance to the antimicrobials. Resistance for cefotaxime and ceftazidime were highest (72-74\%) as compared to the resistance pattern for other antimicrobials. In BSIs, third-generation cephalosporins have been used extensively as a first-line antibiotic, as a result of which they are rendered useless. Our isolates showed least resistance for imipenem and ertapenem, $11.5 \%$, and $26.5 \%$ respectively. The rate of resistance to the various drugs was in concordance with other studies [23, 30,31]. Present study showed good activity of tigecycline (99\%) and colistin (96\%) against the isolates. Only two isolates were found to have intermediate susceptibility to tigecycline. Similar results were documented by Sader et al. [32] and Chen et al. in 2011 [33]. The clinical efficacy of tigecycline in BSI has not yet been established. In vitro, evaluation of its efficacy in ESBL and MBL producing isolates in septicemia have been reported by Roy et al. in two different studies [34, 35].

In the present era, the emergence of MDR organisms and their spread in the community is of great concern. Infections by MDR organisms lead to prolonged hospitalization, increased mortality, morbidity and cost of treatment [36]. As per the definition, MDR in Enterobacteriaceae is defined as "the resistant offered by bacteria to three or more than three antimicrobials of different classes" [37]. Isolates exhibiting co-resistance to at least any three of the following drugs were considered as MDR and these drugs were: extended spectrum cephalosporins (cefixime/ceftriaxone/ceftazidime/cefepime), cephamycins and monobactam or resistant to any two of the above drugs and any one of the carbapenems. In our study, 64 (33.3\%) isolates were found to be MDR. Various authors have reported high percentage of MDR in their study [23,31,38]. Our findings suggest MDR Enterobacteriaceae is less prevalent in our setting as compared to the results of the other studies.

Present study has documented the increasing antimicrobial resistance among isolates from blood stream infections which is the matter of concern for clinicians and microbiologists alike. This reflects the need for early detection and prevention of further spread of resistance to other bacteria.

\section{Conclusion}

Our study has revealed the presence of ESBL, AmpC, carbapenemase and MBL producers in our setting. Occurrence of multidrug resistant Enterobacteriaceae as important etiological agent of BSI is a serious matter of concern. Therefore, it is imperative to take timely steps for the prevention and control of spread of these resistant pathogens. Coordinated efforts from various departments, education of hospital staffs regarding problem of drug resistance, prudent use of antimicrobials and early detection of resistant isolates are required for the achievement of this task. Clinical microbiology laboratory has vital role to play by early and accurate detection of resistant Enterobacteriaceae isolates by ten disk method so that timely action can be taken to curb their spread.

\section{Limitations}

Though molecular techniques are the gold standard, it is difficult for most of the diagnostic laboratories to adopt this test on a routine basis. Though phenotypic methods give little imprecise results, they are widely being used because of their simplicity and cost-effectiveness. $\beta$-lactamase detection by only 


\section{Abbreviations}

\begin{tabular}{|ll|}
\hline AMR & Antimicrobial Resistance \\
\hline ATCC & American Type Culture Collection \\
\hline BSI & Blood Stream Infections \\
\hline CLSI & Clinical and Laboratory Standards Institute \\
\hline DICU & Deluxe Intensive Care Unit \\
\hline ER & Emergency Medicine \\
\hline ESBL & Extended Spectrum Beta-lactamase \\
\hline FDA & Food and Drug Administration \\
\hline GOPD & General Outpatient Department \\
\hline ICU & Intensive Care Unit \\
\hline IPD & Inpatient Department \\
\hline KPC & Klebsiella Pneumoniae Carbapenemase \\
\hline MBL & Metallo Beta-lactamase \\
\hline MDR & Multi Drug Resistant \\
\hline NICU & Neonatal intensive care unit \\
\hline PICU & Pediatric intensive care unit \\
\hline OPD & Outpatient Department \\
\hline ZOI & Zone of Inhibition \\
\hline
\end{tabular}

\section{Declarations}

\subsection{Acknowledgement}

All members of the Department of Microbiology.

\subsection{Funding}

None.

\subsection{Availability of data and materials}

Yes, available.

\subsection{Author's contributions}

Conceptualization: AY, NRB, BK. Investigation: AY. Methodology: AY, NRB, BK. Resources: AY, NRB, BK. Supervision: NRB, BK. Writing-original draft: AY. Writingreview and editing: NRB, BK. All authors read and approved the final manuscript.

\subsection{Ethics approval and consent to participate}

-was obtained from Institutional Review Committee (IRC), BPKIHS, Dharan, Nepal

-code no IRC/424/014

-consent to participate: not applicable

\subsection{Consent for publication}

Not applicable.

\subsection{Competing interest}

We declare that we have no conflict of interest.

\section{References}


1. Bearman GML, Wenzel RP. Bacteremias: a leading cause of death. Arch Med Res. 2005;36(6):646-59.

2. Demirdal ND. and T. Causative agents of nosocomial bloodstream infections and their antimicrobial susceptibility patterns. 2011;5912-6.

3. Ruppé É, Woerther PL, Barbier F. Mechanisms of antimicrobial resistance in Gram-negative bacilli. Ann Intensive Care. 2015;5(1).

4. Bassetti M, Merelli M, Temperoni C, Astilean A. New antibiotics for bad bugs: Where are we? Ann Clin Microbiol Antimicrob. $2013 ; 12(1): 1$.

5. Garcia LS. Clinical Microbiology Procedures handbook. 2nd ed. Washington, DC: ASM press; 2007.

6. Clinical and Laboratory Standards Institute.Performance Standards for Antimicrobial Susceptibility Testing; Twenty-Fourth Informational Supplement. Vol 34. Clinical and Laboratory Standards Institute. Wayne, Pennsylvania, USA; 2014.

7. Singhal S, Mathur T, Khan S, Upadhyay DJ, Chugh S, Gaind R, et al. Evaluation of methods for AmpC beta-lactamase in gram negative clinical isolates from tertiary care hospitals. Indian J Med Microbiol. 2005 Apr;23(2):120-4.

8. Black JA, Moland ES, Thomson KS. AmpC disk test for detection of plasmid-mediated AmpC beta-lactamases in Enterobacteriaceae lacking chromosomal AmpC beta-lactamases. J Clin Microbiol. 2005 Jul;43(7):3110-3.

9. Yong D, Park R, Yum JH, Lee K, Choi EC, Chong Y. Further modification of the Hodge test to screen AmpC $\beta$-lactamase (CMY-1)-producing strains of Escherichia coli and Klebsiella pneumoniae. J Microbiol Methods. 2002 Nov;51(3):407-10.

10. Livermore DM, Winstanley TG, Shannon KP. Interpretative reading: recognizing the unusual and inferring resistance mechanisms from resistance phenotypes. J Antimicrob Chemother. 2001 Jul;48(Suppl 1):87-102.

11. Livermore DM, Brown DF. Detection of beta-lactamase-mediated resistance. J Antimicrob Chemother. 2001 Jul;48(Suppl 1):59-64.

12. Thomson KS. Extended-spectrum-beta-lactamase, AmpC, and Carbapenemase issues. J Clin Microbiol. 2010 Apr;48(4):1019-25.

13. Franklin C, Liolios L, Peleg AY. Phenotypic detection of carbapenem-susceptible metallo- $\beta$-lactamase- producing gram-negative bacilli in the clinical laboratory. J Clin Microbiol. 2006;44(9):3139-44.

14. Lee K, Lim YS, Yong D, Yum JH, Chong Y. Evaluation of the Hodge test and the imipenem-EDTA double-disk synergy test for differentiating metallo-betalactamase-producing isolates of Pseudomonas spp. and Acinetobacter spp. J Clin Microbiol. 2003 Oct;41(10):4623-9.

15. Anderson KF, Lonsway DR, Rasheed JK, Biddle J, Jensen B, McDougal LK, et al. Evaluation of methods to identify the Klebsiella pneumoniae carbapenemase in Enterobacteriaceae. J Clin Microbiol. 2007 Aug;45(8):2723-5.

16. Datta S, Wattal C, Goel N, Oberoi JK, Raveendran R, Prasad KJ. A ten year analysis of multi-drug resistant blood stream infections caused by Escherichia coli \& Klebsiella pneumoniae in a tertiary care hospital. Indian J Med Res. 2012 Jun;135(6):907-12.

17. Abdallah HM, Wintermans BB, Reuland EA, Koek A, al Naiemi N, Ammar AM, et al. Extended-Spectrum $\beta$-Lactamase- and Carbapenemase-Producing Enterobacteriaceae Isolated from Egyptian Patients with Suspected Blood Stream Infection. PLoS One. 2015 Jan;10(5):e0128120.

18. Joshy M, Easow, Noyal M, Joseph BA, Dhungel, Bipin Chapagain PS. Blood Stream Infections among febrile patients attending a Teaching Hospital in Western Region of Nepal. Australasian Medical Journal AMJ. 2010;3:10, 633-7.

19. Falagas ME, Bliziotis IA. Pandrug-resistant Gram-negative bacteria: the dawn of the post-antibiotic era? Int J Antimicrob Agents. 2007 Jun;29(6):630-6.

20. Shrestha S, Amatya R, Dutta R. Prevalence of extended spectrum beta lactamase (ESBL) production in gram negative isolates from pyogenic infection in tertiary care hospital of eastern Nepal. Nepal Med Coll J. 2011 Sep;13(3):186-9.

21. Raut S, Gokhale S, Adhikari B. Prevalence of Extended Spectrum Beta-Lactamases among Escherichia coli and Klebsiella spp isolates in Manipal Teaching Hospital, Pokhara. J Microbiol Infect Dis. 2015.

22. Poudyal S, Bhatta DR, Shakya G, Upadhyaya B, Dumre SP, Buda G, et al. Extended spectrum â-lactamase producing multidrug resistant clinical bacterial isolates at National Public Health Laboratory, Nepal. Nepal Med Coll J. 2011 Mar;13(1):34-8.

23. Modi Dhara J, Patel Bhaumik V, Patel Mitesh H, Bhatt Seema S, Sood Nidhi KV. M M. A study of extended spectrum $\beta$-lactamase (ESBL) and AmpC $\beta$ lactamase producing Klebsiella pneumoniae in neonatal intensive care unit at tertiary care hospital, Ahmedabad. Natl J Community Med. 2012.

24. Baral P, Neupane S, Shrestha B, Ghimire KR, Marasini BP, Lekhak B. Clinical and microbiological observational study on AmpC $\beta$-lactamase-producing Enterobacteriaceae in a hospital of Nepal. Braz J Infect Dis. 2013 Jan;17(2):256-9.

25. Carter MW, Oakton KJ, Warner M, Livermore DM. Detection of extended-spectrum beta-lactamases in klebsiellae with the Oxoid combination disk method. J Clin Microbiol. 2000 Nov;38(11):4228-32.

26. Granier SA, Leflon-Guibout V, Nicolas-Chanoine M-H, Bush K, Goldstein FW. The extended-spectrum K1 beta-lactamase from Klebsiella oxytoca SC 10,436 is a member of the bla(OXY-2) family of chromosomal Klebsiella enzymes. Antimicrob Agents Chemother. 2002 Jun;46(6):2056-7.

27. Castanheira M, Sader HS, Deshpande LM, Fritsche TR, Jones RN. Antimicrobial activities of tigecycline and other broad-spectrum antimicrobials tested against serine carbapenemase- and metallo-beta-lactamase-producing Enterobacteriaceae: report from the SENTRY Antimicrobial Surveillance Program. Antimicrob Agents Chemother. 2008 Feb;1(2):570-3. 52.

28. Bogdanovich T, Adams-Haduch JM, Tian G-B, Nguyen MH, Kwak EJ, Muto CA, et al. Colistin-resistant, Klebsiella pneumoniae carbapenemase (KPC)producing Klebsiella pneumoniae belonging to the international epidemic clone ST258. Clin Infect Dis. 2011 Aug;53(4):373-6.

29. Mishra SK, Shrestha R, Rijal BP, Pokhrel BM. The bad, the ugly and the demon: a tale of extensively drug-resistant, extended-spectrum-beta-lactamase- and metallo-beta-lactamase-producing superbugs associated with nosocomial pneumonia. Asian Pacific J Trop Dis. 2015 Jan;5(1):71-6.

30. Gajul SV, Mohite ST, Mangalgi SS, Wavare SM, Kakade SV. Klebsiella Pneumoniae in Septicemic Neonates with Special Reference to Extended Spectrum ß-lactamase, AmpC, Metallo $\beta$-lactamase Production and Multiple Drug Resistance in Tertiary Care Hospital. J Lab Physicians. 2015 Jan;7(1):32-7.

31. Chandel DS, Johnson JA, Chaudhry R, Sharma N, Shinkre N, Parida S, et al. Extended-spectrum beta-lactamase-producing Gram-negative bacteria causing neonatal sepsis in India in rural and urban settings. J Med Microbiol. 2011 Apr;60(Pt 4):500-7.

Page $9 / 12$ 
32. Sader HS, Farrell DJ, Jones RN. Tigecycline activity tested against multidrug-resistant Enterobacteriaceae and Acinetobacter spp. isolated in US medical centers (2005-2009). Diagn Microbiol Infect Dis. 2011 Feb;69(2):223-7.

33. Chen S, Hu F, Zhang X, Xu X, Liu Y, Zhu D, et al. Independent emergence of colistin-resistant Enterobacteriaceae clinical isolates without colistin treatment. J Clin Microbiol. 2011 Nov;49(11):4022-3.

34. Roy S, Datta S, Viswanathan R, Singh AK, Basu S. Tigecycline susceptibility in Klebsiella pneumoniae and Escherichia coli causing neonatal septicaemia (2007-10) and role of an efflux pump in tigecycline non-susceptibility. J Antimicrob Chemother. 2013 May;68(5):1036-42.

35. Roy S, Viswanathan R, Singh AK, Das P, Basu S. Sepsis in neonates due to imipenem-resistant Klebsiella pneumoniae producing NDM-1 in India. J Antimicrob Chemother. 2011 Jun;66(6):1411-3.

36. Behera B, Das A, Mathur P, Kapil A, Gadepalli R, Dhawan B. Tigecycline susceptibility report from an Indian tertiary care hospital. Indian J Med Res. 2009 Apr;129(4):446-50.

37. Magiorakos A-P, Srinivasan A, Carey RB, Carmeli Y, Falagas ME, Giske CG, et al. Multidrug-resistant, extensively drug-resistant and pandrug-resistant bacteria: an international expert proposal for interim standard definitions for acquired resistance. Clin Microbiol Infect. 2012 Mar;18(3):268-81.

38. VinodKumar CS, Kalappanavar NK, Umakanth Patil BKG. Change in spectrum of microbial aetiology in relation to gestational age and birth weight and emergence of ESBL in tertiary neonatal intensive car. Int J Biol Med Res. 2011;2(3):727-34.

\section{Figures}

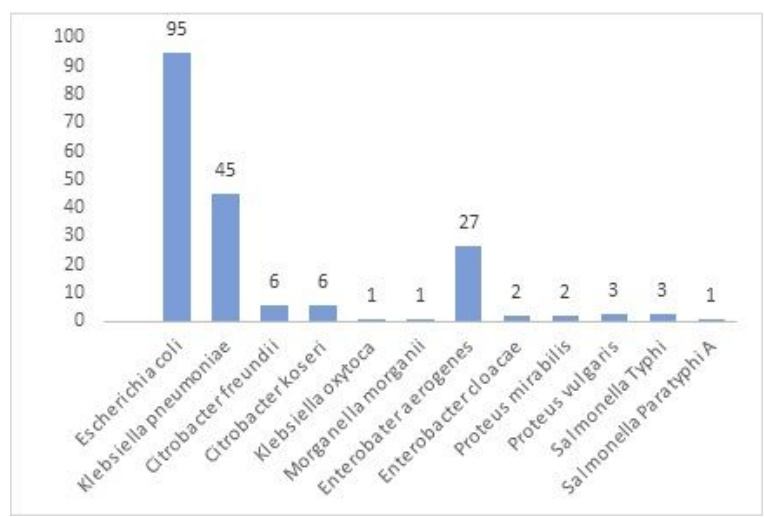

\section{Figure 1}

Frequency of Enterobacteriaceae isolates $(n=192)$

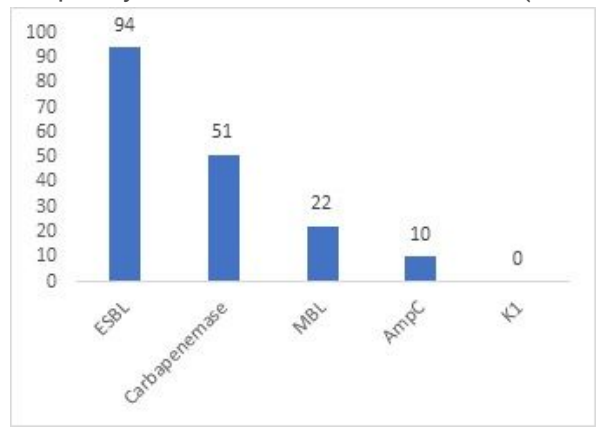

Figure 2

$\beta$-lactamase producers $(n=192)$ 


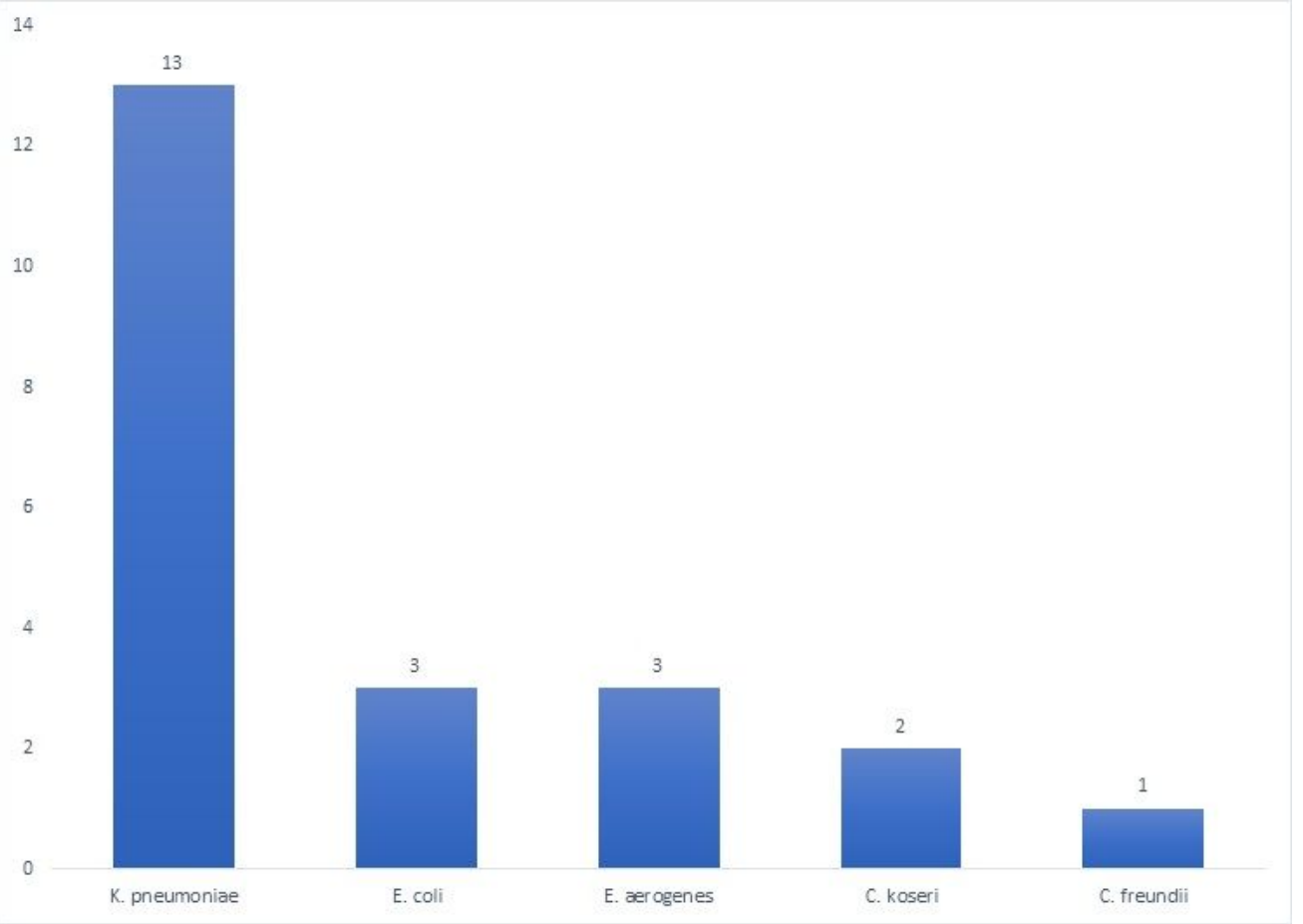

Figure 3

MBL producers $(n=22)$

30

26

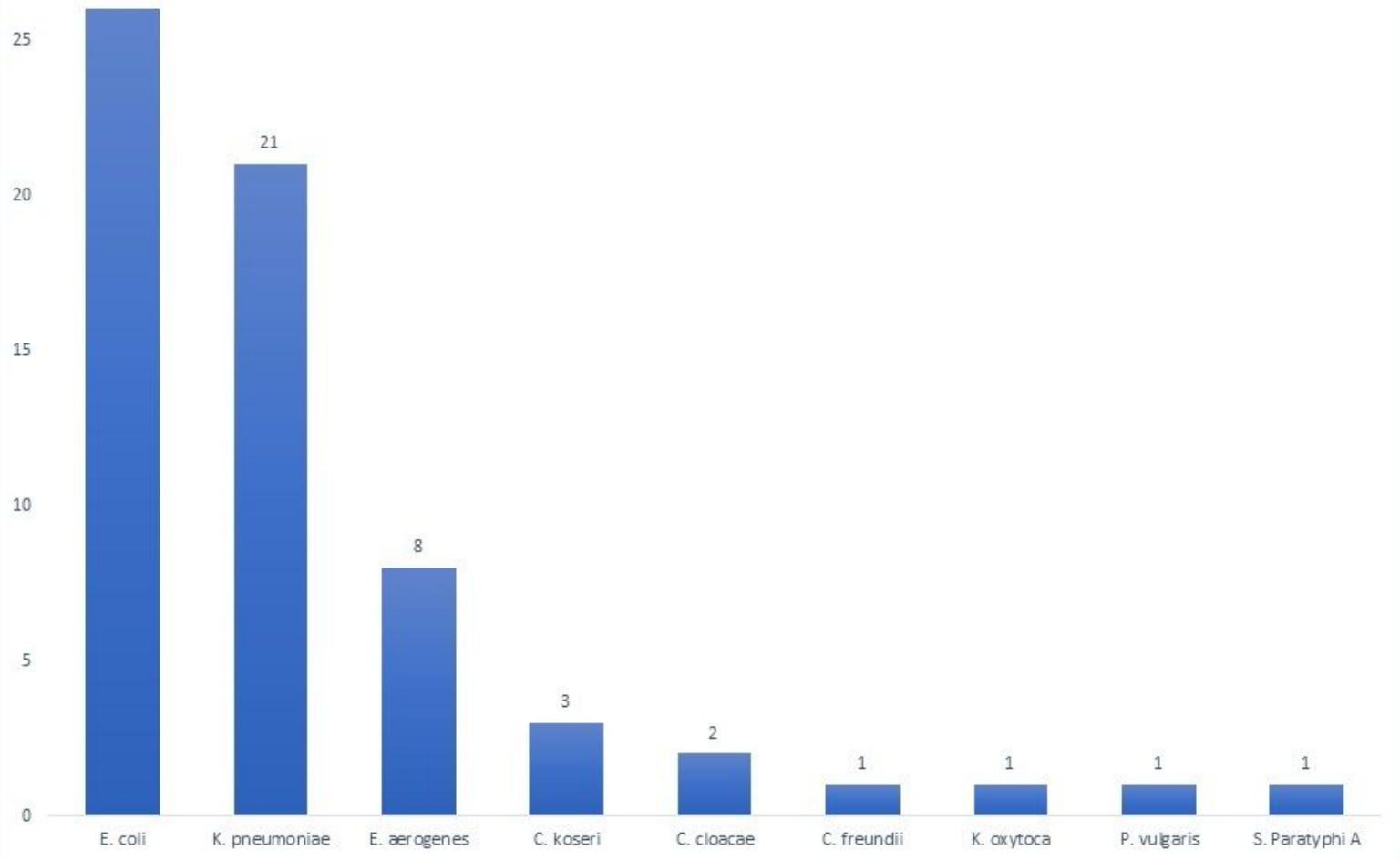

Figure 4

MDR Enterobacteriaceae $(n=64)$ 
12

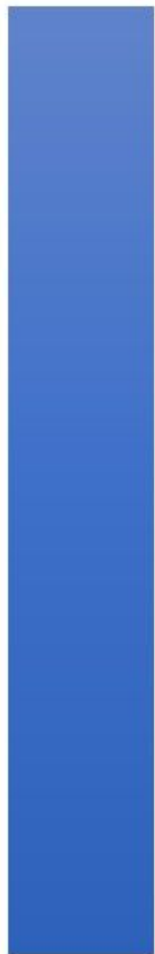

K. pneumoniae

\section{Figure 5}

$\beta$-lactam drugs resistant Enterobacteriaceae $(n=22)$ 